tion of the same quantum defect for Be III, B IV, and $\mathrm{C} \mathrm{V}$ gives the following term values:

\begin{tabular}{|c|c|c|}
\hline & B IV. & C v. \\
\hline$n-n^{*}$ & $-0 \cdot 013 \pm 0 \cdot 002$ & $-0 \cdot 013 \pm 0 \cdot 003$ \\
$2^{1} P$ & $433 \cdot 300 \pm 900 \mathrm{~cm} .^{-1}$ & $677,000 \pm 2000 \mathrm{~cm}^{-1}$ \\
$3^{1} P$ & $193 \cdot 400 \pm 300 \quad$, & \\
$1^{1} S$ & $2091 \cdot 500 \pm 1400 \quad$, & $3,159,600 \pm 3300 \quad$, \\
\hline
\end{tabular}

The ionising potentials, $1^{1} S \times 1.234 \times 10^{-4}$, are in good agreement with the quantum theoretical values given by Hylleraas ${ }^{4}$ in the formula

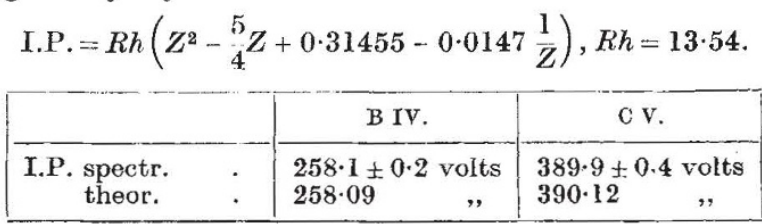

It is hoped that the accuracy will be considerably increased by measurements in higher orders and a more confident determination of the ${ }^{1} P$ terms.

From the resonance line of $\mathrm{C} \mathrm{V}$ at $40 \mathrm{~A}$. the region is completely bare of carbon lines up to $200 \mathrm{~A}$., where the principal series $2^{2} S-n^{2} P$ of C IV forms the limit of the $L$-electron spectrum. Then, including spark spectra of sufficiently high orders, the wide separation between the $K$ and $L$ spectra, typical for the $\mathrm{X}$-rays, will also appear in optical spectra.

Physics Laboratory, University, BengT EDLÉN. Uppsala, Feb. 1.

1 B. Edlén and A. Ericson, Natore, 125, 233; 1930 ; and Zeit.f. Phys, 59,$658 ; 1930$.

w. Ekeforg, Physik. Zeitschr., 31, $737 ; 1930$.

4 E. A. Hyllerags,

\section{Fluorescence of Mercury Vapour under Atomic and Molecular Absorption.}

IN the letter which appears in NaTure of Jan. 3, p. 10, Lord Rayleigh discusses the excitation of the green fluorescence of mercury vapour by wave-lengths near the resonance line $\lambda 2537$. The discontinuity of intensity of the fluorescent radiation along the beam of the exciting light leads him to admit the atomic as well as the molecular absorption. The hypothesis of two kinds of absorption exciting the fluorescence was further confirmed by Lord Rayleigh's investigation of the influence of the hydrogen admixture. These experiments support the view that the fluorescence of mercury vapour is chiefly due to atomic absorption.

I should like to point out that this conclusion agrees with that which $I$ have drawn from my experiments on the influence of the magnetic field on the fluorescence of mercury vapour. ${ }^{1}$ In these experiments the fluorescence was excited by the light of the mercury line $\lambda 2537$. The tube with the distilling mercury vapour was placed between the poles of a powerful electromagnet and the intensity of the fluorescent radiation was observed at different strengths of the magnetic field as measured at the place of excitation. The variation of the intensity of the green fluorescence was found similar to that of the resonance radiation investigated by M. Schein. ${ }^{2}$ The maxima and minima of the intensity of both radiations appeared at equal magnetic fields, thus indicating that the region of the excitation of the fluorescence has exactly the same hyperfine structure as the exciting line $\lambda 2537$. Hence the only possible conclusion seemed to be that, in the excitation of the fluorescence of mercury vapour at moderate densities by the line $\lambda 2537$, the atomic absorption forms the first necessary step of the excitation.

The evidence of my experiments referred to above and the recent work of Lord Rayleigh are decidedly against the view often expressed that the fluorescence of the mercury vapour should be produced by the molecular absorption alone.

HENRYK NIEWODNICZAŃSKr.

Stefan Batory University,

Wilno, Poland, Jan. 30.

I Zeitschrift für Physik, vol. 55, p. $676 ; 1929$.
2 Annalen der Physik, vol. 85, p. 257; 1928.

\section{Raman Spectrum of Hydrogen Peroxide.}

THE Raman spectrum of hydrogen peroxide (Merck's perhydrol 30 per cent solution in water) has been photographed. The prominent feature of the spectrum is the presence of a sharp Raman frequency of 875 accompanied by a weak component at 903 . The low value of the frequencies suggests that they arise from the oxygen atoms, and as such may be compared with the $\mathrm{O}_{2}$ oscillation frequency 1552 obtained by McLennan (Trans. Farad. Soc., 25, 798 ; 1929). The large difference between the two values is indicative of the fact that the oxygen atoms in hydrogen peroxide $\left(\mathrm{H}_{2} \mathrm{O}_{2}\right)$ and oxygen $\left(\mathrm{O}_{2}\right)$ molecules respectively are bound differently. Besides the above two frequencies, there appear other bands which are less intense and sharp, the origin of which is under investigation. A detailed discussion of the results obtained in relation to the structure of the molecule will appear elsewhere.

S. VENKATESWARAN.

210 Bow Bazar Street

Calcutta, Jan. 17.

Resistance of Eurytemora hirundoides Nordquist, a Brackish Water Copepod, to Oxygen Depletion.

The River Tyne estuary is polluted by crude sewage and manufacturing effluents to such an extent as to cause a serious depletion in dissolved oxygen. It is not unusual to find a complete absence of dissolved oxygen on isolated occasions during the summer months. The number of normal estuarine organisms able to withstand these adverse conditions is very limited. 1 Of these, Eurytemora hirundoides is the most abundant and the most hardy. Its region of maximum abundance lies between 9 miles and 13 miles from the river mouth, in the area of heaviest pollution and most noticeable oxygen depletion. At a survey held on July 22, 1925, it occurred plentifully in regions where the dissolved oxygen was nil, as determined by the Winkler method.1,2 During 1929 I obtained it freely in the water sampler on occasions when the oxygen content was again nil (these remarks apply to the sampling depth of $6 \mathrm{ft}$.).

There are, at the present time, several investigations being made into the oxygen requirements of certain marine animals. It may, therefore, be of interest to place on record the following additional observations upon Eurytemora, made incidentally in the course of other work.

In addition to determining the dissolved oxygen in situ of the River Tyne at Newcastle, I have for the past two years included an additional test described as the 'dissolved oxygen absorbed'. This is a modification by Jackson and Jee of the test recommended by the Royal Commission on Sewage Disposal. ${ }^{3}$ A stoppered bottle of approximately 330 c.c. capacity is filled with the river water (taking the usual preeautions to exclude all air) and 'incubated ' by complete immersion in water at air temperature for a period of seven days. The oxygen remaining is de-

No. 3202 , VoL. 1277 